\title{
Analysis of Main Characteristics of Hydro-mechanical Continuously Variable Transmission
}

\author{
Zengming Zhang ${ }^{1}$, Huanyong Cui ${ }^{1,{ }^{*}}$, Ruichuan $\mathrm{Li}^{2}$, Xijie $\mathrm{Tian}^{1}$, Xinghua $\mathrm{Hu}^{1}$ and \\ Jikang $\mathrm{Xu}^{3}$ \\ ${ }^{1}$ School of mechanical engineering, University of Jinan, Jinan 250022, China \\ ${ }^{2}$ Wuzheng Group, Rizhao 276800, China \\ ${ }^{3}$ Rizhao Haizhuo Hydraulic Co., Ltd, Rizhao 276800, China
}

\begin{abstract}
Keywords: Power Split, Hydro-mechanical Continuously Variable Transmission, Characteristics Analysis.
\end{abstract}

\begin{abstract}
A kind of hydro-mechanical continuously variable transmission (HMCVT) is put forward on the basis of the power split fundamental principle. The equations of speed, torque, power and efficiency characteristics of HMCVT are established according to the transmission principle in this paper. The analysis results show that HMCVT has the function of steepless transmission by the control of the hydraulic system; the adoption of planets and gear shift mechanisms contributes to expanding the variable speed range of HMCVT; the HMCVT could satisfy the operation requirements of the high-power tractors with the characteristic that the large output torque at the low speed range and high efficiency at most of speed range.
\end{abstract}

\section{Introduction}

The hydro-mechanical continuously variable transmission (HMCVT), which is a special power split transmission with characteristics of variable ratio and high efficiency, is suitability for high-power tractors and heavy vehicles [1]. During a few decades, many products of HMCVT have been developed and applied in high-power tractors in Europe and America. The first HMCVT installed in tractors was Fent's Vario in 1996. Then the Steyr Case's S-matic, ZF's Ecoom, John Deere's Autopower were brought out one by one [2,3]. The demand of HMCVT will be increasingly more in high-power tractors under the rapid development of modern and intelligent agriculture. In this paper, a transmission scheme of HMCVT is proposed with the analysis of transmission principle and working characteristic which will provide theoretical basis for HMCVT scheme design, parameter matching and performance analysis.

\section{Transmission Principle of HMCVT}

The hydro-mechanical continuously variable transmission (HMCVT), a kind of power split transmission, will split the input power into two parts, one is the mechanical path with fixed transmission ratio, and another is hydrostatic path with variable transmission ratio; the power of two parts will be summed up in the output shaft by planetary gear or fixed gears [4, 7]. Fig. 1 shows an input coupled type transmission with a variable pump-quantitative motor system. It will get a variable transmission ratio $i$ by changing the displacement ratio $e$ as shown in Fig. 2. The transmission ratio $i$ represents the ratio of output speed and the input speed; the displacement ratio $e$ represents the ratio of the pump displacement and the motor displacement.

The scheme of HMCVT shown in Fig.3 is established on the basis of the transmission principle of the power split fundamental principle. The HMCVT is mainly composed of the variable pump-quantitative motor system, double row planetary and several gear shift mechanisms with 5 forward working sections (H, HM1, HM2, HM3 and HM4) and 5 backward working sections (R1, R2, 
R3, R4 and R5).

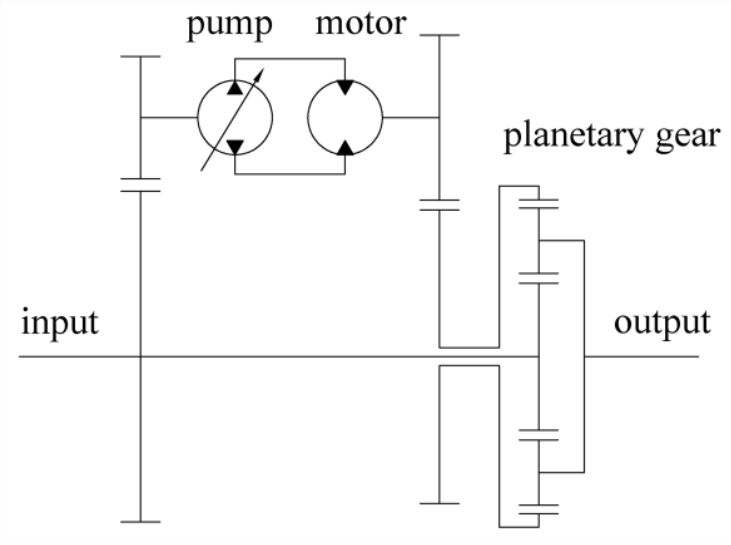

Figure 1. Input coupled type transmission

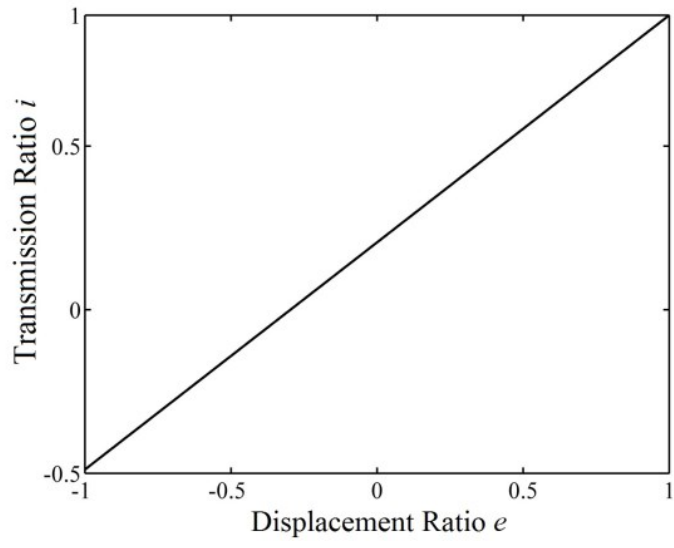

Figure 2. Relationship between i and e

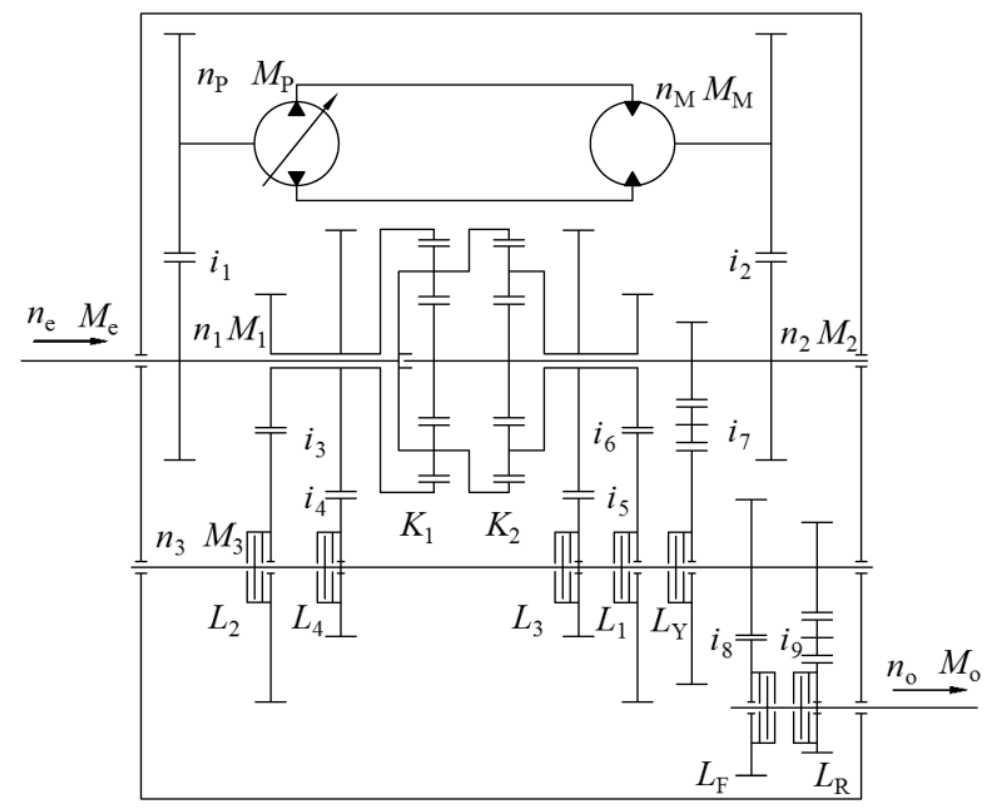

Figure 3. Transmission scheme of the HMCVT

Different working sections of HMCVT are controlled by clutches (LY、L1、L2、L3、L4、L5、 LF and LR) as shown in Table 1.

Table 1 Engagement status of clutches

\begin{tabular}{|c|c|c|c|c|c|c|c|c|}
\hline \multirow{2}{*}{ Work section } & \multicolumn{7}{|c|}{ Engagement status of clutches } \\
\cline { 2 - 10 } & LY & L1 & L2 & L3 & L4 & LF & LR \\
\hline \multirow{5}{*}{ Forward } & H & $\bullet$ & & & & & $\bullet$ & \\
\cline { 2 - 9 } & HM1 & & $\bullet$ & & & & $\bullet$ & \\
\cline { 2 - 9 } & HM2 & & & $\bullet$ & & & $\bullet$ & \\
\cline { 2 - 9 } & HM3 & & & & $\bullet$ & & $\bullet$ & \\
\cline { 2 - 9 } & HM4 & & & & & $\bullet$ & $\bullet$ & \\
\hline \multirow{5}{*}{ Backward } & R1 & $\bullet$ & & & & & & $\bullet$ \\
\cline { 2 - 9 } & R2 & & $\bullet$ & & & & & $\bullet$ \\
\cline { 2 - 9 } & R3 & & & $\bullet$ & & & & $\bullet$ \\
\cline { 2 - 9 } & R4 & & & & $\bullet$ & & & $\bullet$ \\
\cline { 2 - 9 } & R5 & & & & & $\bullet$ & & $\bullet$ \\
\hline
\end{tabular}

Table 2. Transmission ratio of HMCVT

\begin{tabular}{|c|c|c|c|}
\hline \multicolumn{2}{|c|}{ Working section } & $i$ & $e$ \\
\hline \multirow{7}{*}{ Forward } & $\mathrm{H}$ & $i=-\frac{e}{i_{1} i_{2} i_{7} i_{8}}$ & $0 \sim-1$ \\
\cline { 2 - 4 } & HM1 & $i=\frac{e+k_{2} i_{1} i_{2}}{\left(1+k_{2}\right) i_{1} i_{2} i_{6} i_{8}}$ & $-1 \sim+1$ \\
\cline { 2 - 4 } & HM2 & $i=\frac{i_{1} i_{2}\left(1+k_{1}\right)-e}{k_{1} i_{1} i_{2} i_{3} i_{8}}$ & $+1 \sim-1$ \\
\cline { 2 - 4 } & HM3 & $i=\frac{e+k_{2} i_{1} i_{2}}{\left(1+k_{2}\right) i_{1} i_{2} i_{5} i_{8}}$ & $-1 \sim+1$ \\
\cline { 2 - 4 } & HM4 & $i=\frac{i_{1} i_{2}\left(1+k_{1}\right)-e}{k_{1} i_{1} i_{2} i_{4} i_{8}}$ & $+1 \sim-1$ \\
\hline
\end{tabular}

In the Table 1, “ ” stands for the engagement of a clutch, "H” and "HM" express respectively 
the pure hydraulic transmission section and the hydraulic-mechanical transmission section, "R" represents the reverse gear section.

\section{Speed characteristics of HMCVT}

The speed characteristic is defined as the relationship between the transmission ratio $i$ and the displacement ratio $e$ [5].According to the transmission principle of HMCVT, the speed matrix equation of the components in HM1 can be derived as the Eq. 1:

$$
\left[\begin{array}{cccccccc}
1 & -i_{1} & 0 & 0 & 0 & 0 & 0 & 0 \\
0 & 1 & -e & 0 & 0 & 0 & 0 & 0 \\
0 & 0 & 1 & -i_{2} & 0 & 0 & 0 & 0 \\
k_{2} & 0 & 0 & 1 & -\left(1+k_{2}\right) & 0 & 0 & 0 \\
-\left(1+k_{2}\right) & 0 & 0 & 1 & 0 & k_{2} & 0 & 0 \\
0 & 0 & 0 & 0 & 1 & -i_{6} & 0 & 0 \\
0 & 0 & 0 & 0 & 0 & 0 & 1 & -i_{8}
\end{array}\right]\left(\begin{array}{c}
n_{1} \\
n_{P} \\
n_{M} \\
n_{2} \\
n_{c 2} \\
n_{r 1} \\
n_{3} \\
n_{o}
\end{array}\right)=0
$$

where: $i_{1}, i_{2}, \ldots, i_{9}$ are the ratio of fixed gears; $k_{1}$ and $k_{2}$ represent respectively the characteristic parameters of the planetary gears $\mathrm{K} 1$ and $\mathrm{K} 2 ; n_{\mathrm{s}}, n_{\mathrm{r}}$ and $n_{\mathrm{c}}$ represent respectively the rotational speeds of sun gear, ring gear and carrier of the planetary gear.

According to the Eq. 1, the transmission ratio $i$ can be derived as the Eq. 2. The transmission ratio $i$ of the other forward working sections are shown in table 2 .

$$
i=\frac{n_{o}}{n_{1}}=\frac{e+k_{2} i_{1} i_{2}}{\left(1+k_{2}\right) i_{1} i_{2} i_{6} i_{8}}
$$

As shown in the Table 2, $k_{1}, k_{2}$ and the ratio of fixed gears are constants which will influence the transmission ratio of HMCVT. It's continuous and linear relationship between the transmission ratio $i$ of each work section and the displacement ratio with the other constant parameters. The transformation between adjacent work sections should fulfill the working conditions: (1) the equal transmission ratio $i$ between the adjacent work sections; (2) the equal displacement ratio $e$ between the adjacent work sections.

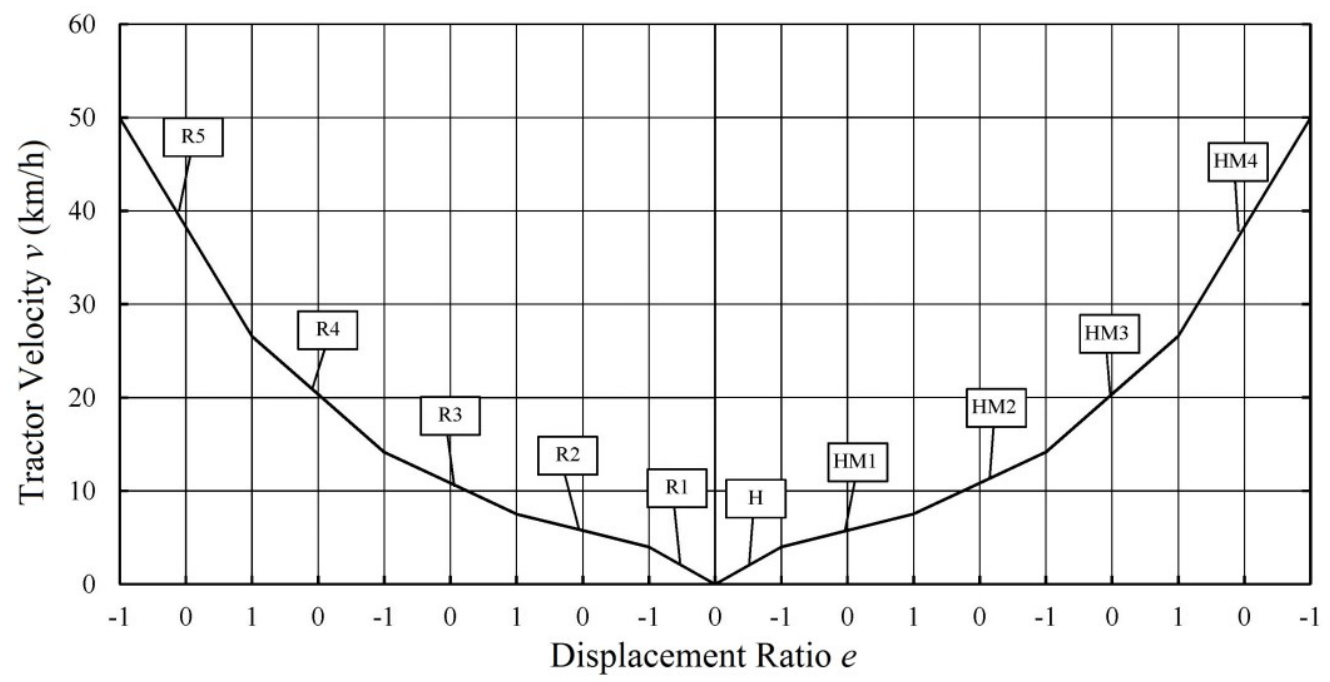

Figure 4. Characteristic curve of speed regulation of HMCVT

The relationship of the tractor speed $v$ and the displacement ratio $e$ is shown in Fig. 4 . The HMCVT can achieve the speed in the range of $0 \sim 50 \mathrm{~km} / \mathrm{h}$ used for 200 horsepower tractors. 


\section{Torque characteristics of HMCVT}

In the HMCVT, the maximum output torque of HMCVT $M_{\text {omax }}$ depends on the maximum output torque of the quantitative motor $\mathrm{M}_{\operatorname{mmax}}$ [1]. So the torque characteristic of HMCVT is defined as the relationship between $M_{\mathrm{omax}}$ and $\mathrm{M}_{\operatorname{mmax}}$.

The expressions of the maximum output torque of the forward work section are derived in the Table 3 according to the transmission principle of HMCVT. And the torque characteristics curve of the forward work section is shown in the Fig. 5.

Table 3. Maximum output torque of HMCVT

\begin{tabular}{|c|c|c|}
\hline \multicolumn{2}{|c|}{ Working section } & Maximum output torque Momax \\
\hline \multirow{4}{*}{ Forward } & $\mathrm{H}$ & $M_{\text {omax }}=i_{2} i_{7} i_{8} M_{\text {mmax }}$ \\
\cline { 2 - 3 } & $\mathrm{H} 1$ & $M_{\text {omax }}=\left(1+k_{2}\right) i_{2} i_{6} i_{8} M_{\operatorname{mmax}}$ \\
\cline { 2 - 3 } & $\mathrm{H} 2$ & $M_{\text {omax }}=k_{1} i_{2} i_{3} i_{8} M_{\text {mmax }}$ \\
\cline { 2 - 3 } & HM3 & $M_{\text {omax }}=\left(1+k_{2}\right) i_{2} i_{5} i_{8} M_{\text {mmax }}$ \\
\cline { 2 - 3 } & HM4 & $M_{\text {omax }}=k_{1} i_{2} i_{4} i_{8} M_{\text {mmax }}$ \\
\hline
\end{tabular}

Table 4. Hydraulic power split ratio $\rho$ of HMCVT

\begin{tabular}{|c|c|c|}
\hline \multicolumn{2}{|c|}{ Working section } & Hydraulic power split ratio $\rho$ \\
\hline \multirow{4}{*}{ Forward } & $\mathrm{H}$ & $\rho=1$ \\
\cline { 2 - 3 } & HM1, HM3 & $\rho=\frac{e}{e+k_{2} i_{1} i_{2}}$ \\
\cline { 2 - 3 } & HM2 ,HM4 & $\rho=\frac{e}{e-i_{1} i_{2}\left(1+k_{1}\right)}$ \\
\hline
\end{tabular}

The pure hydraulic transmission section $\mathrm{H}$ is used for the smooth starting of vehicle with a small torque. The torques of the forward work sections decrease progressively from HM1 section to HM4 section. The HMCVT enables a tractor to have the better accelerating ability with the large output torque in the low speed range.

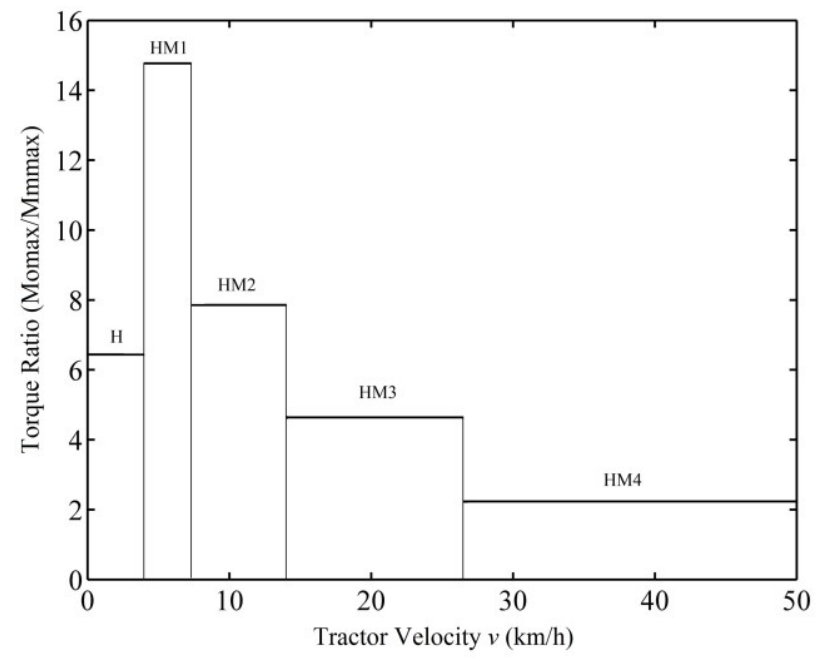

Figure 5. Torque characteristics of HMCVT

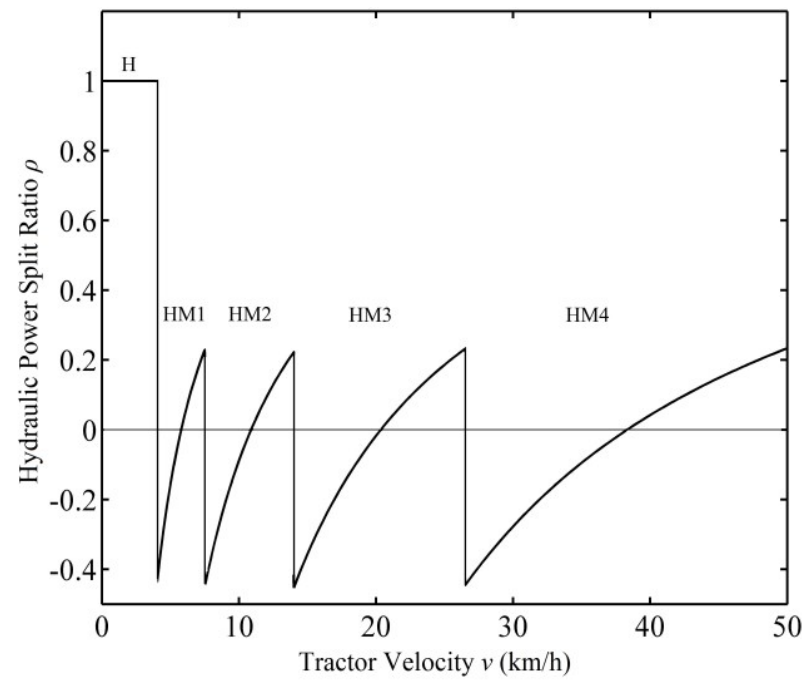

Figure 6. Hydraulic power split ratio $\rho$ of HMCVT

\section{Power and efficiency characteristics of HMCVT}

In the HMCVT, the ratio of the output power of the hydraulic branch and the output power of HMCVT is defined as the hydraulic power split ratio $\rho$ [6]. The small hydraulic power split ratio $\rho$ will be conducive to improving the transmission efficiency of HMCVT because of the high mechanical efficiency.

The $\mathrm{H}$ working section is the pure hydraulic transmission without power transmitting in mechanical path. The hydraulic power split ratio in H working section: $\rho=1$. 
The HM1 section is the hydraulic-mechanical transmission. The hydraulic power split ratio $\rho$ can be obtained based on the HMCVT working principle as in Eq.3. The other parts of the hydraulic power split ratios as shown in Table 4.

$$
\rho=-\frac{M_{M} n_{M}}{M_{o} n_{o}}=\frac{e}{e+k_{2} i_{1} i_{2}}
$$

The power split ratio curve of each work section was drawn in the Fig. 6. Most of the hydraulic power split ratio $\rho$ is less than 0.3 in the whole tractor speed range with low power loss generated by the hydraulic transmission which will make the HMCVT have higher transmission efficiency about $0.85 \sim 0.95$.

\section{Summary}

This paper has derived the equations of speed, torque, power and efficiency characteristics of the HMCVT. In the HMCVT, planetary parameters, fixed gears ratio and displacement ratio are the main parameters that influence the transmission characteristics. A low the hydraulic power split ratio $\rho$ will improve the system transmission efficiency. The variable speed range of HMCVT can be enlarged by the use of step speed changing mechanism which is controlled by clutches. But it must meet the two working conditions for transforming between each work sections.

\section{Acknowledgement}

The authors would like to acknowledge the financial support by Jinan Municiple Science\&Technology Developing Plan (201118001) and Shandong Provincial Science\&Technology Developing Plan (2012GGX10302).

\section{References}

[1] Xiuji Liu, Analysis of Vehicle transmission system, first ed., National Defence Industry Press, Beijing, 1998.

[2] P. Linares, V. Méndez, H. Catalán, Design parameters for continuously variable power-split transmissions using planetaries with 3 active shafts, J. Journal of Terramechanics. 47(2010) 323-335.

[3] K.T. Renius, R. Resch, Continuously variable tractor transmissions, in: ASAE distinguished lecture series, No. 29, 2005.

[4] A. Rossetti, A. Macor, Multi-objective optimization of hydro-mechanical power split transmissions, J. Mechanism \& Machine Theory. 62(2013) 112-128.

[5] Liyou Xu, Zhili Zhou, Mingzhu Zhang, Characteristics analysis of hydro-mechanical continuously variable transmission of tractor, J. Journal of China Agricultural University.11(2006)70-74.

[6] Jingbo Guo, Tiaoquan Lai, Characteristics analysis of the diversion ratio and transmission of hydro-mechanical composite transmission, J. Journal of Construction Machinery, 10(1997): 38 41, 47.

[7] A. Rossetti, A. Macor, Multi-objective optimization of hydro-mechanical power split transmissions, J. Mechanism \& Machine Theory. 62(2013) 112-128. 